\section{Avaliação do Curso de Saúde e Seguran- ça do Trabalho - Capacitação segundo a NR32: Campus de Ribeirão Preto / USP de 2010 a 2013}

\author{
Evaluation of Course on Work Health and Safety - Training \\ according to NR32: Ribeirão Preto Campus / USP from \\ 2010 to 2013
}

\section{RESUMO}

Os dados de avaliação do curso Saúde e Segurança do Trabalho - capacitação segundo a NR32, oferecido anualmente, no período de 2010 a 2013, no Campus da USP de Ribeirão Preto são apresentados neste trabalho. $\mathrm{O}$ objetivo do curso é promover a capacitação de servidores não docentes em saúde e segurança no trabalho ao propiciar condições para minimização de riscos de diferentes agentes nos ambientes ocupacionais por meio da aquisição de conhecimentos a respeito das doenças, acidentes e normas vigentes. Durante os quatro anos do curso predominou a participação do gênero feminino, sendo a Faculdade de Medicina de Ribeirão Preto, a unidade com maior número de alunos. Quanto à atividade funcional dos alunos, houve predomínio daqueles provenientes de biotério e de laboratórios, especialmente de técnicos e auxiliares. Assim, pela contínua demanda e resultado constatado ao longo do período analisado, considera-se que o curso alcançou seus objetivos, enquanto módulo de educação continuada, o que indica a necessidade da manutenção de seu oferecimento anual. Ainda, nos próximos anos almeja-se avaliar o impacto do curso nas unidades e serviços do campus, visando o aprimoramento e adequação às reais necessidades dos diferentes setores de atuação dos servidores do campus USP de Ribeirão Preto.

Pallavras-chave: Saúde Ocupacional. Segurança no Trabalho. Qualidade de Vida.

\section{ABSTRACT}

Evaluation data from the course Work Health and Safety - Training according to $\mathrm{NR}_{32}$, offered annually in the period 2010-2013 are presented in this paper. The aim of this course is to promote work health and safety training non-professors to provide conditions for minimizing risks of different agents in occupational settings through the acquisition of knowledge about the diseases, accidents and current standards. During four years of the course, the predominant participation of females, being Ribeirão
EVANDRO WATANABE E Ana Maria Razaboni Universidade de São Paulo. Faculdade de Odontologia de Ribeirão Preto, São Paulo, Brasil

Angela Maria

MAgOSSO TAKAYANAgU I

Universidade de São Paulo. Escola de Enfermagem de Ribeirão Preto, São Paulo, Brasil

Alcyone Artioli MaCHADO

Universidade de São Paulo. Faculdade de Medicina de Ribeirão Preto, São Paulo, Brasil

\section{Sandra Márcia de} Castro

Universidade de São Paulo. Serviço Especializado em Engenharia de Segurança e Medicina do Trabalho de Ribeirão Preto, São Paulo, Brasil 
Preto Medical School, the unit with the largest number of students. Regarding the functional activity of the students were predominantly those from biotherium and laboratory, especially technicians and assistants. Thus, the continuous demand and result observed over the period analyzed, it is considered that the course has achieved its objectives, while continuing education module, which indicates the necessity of maintaining its annual offering. Besides, in the coming years we aim to evaluate the impact of the course in the units and campus services, aiming at the improvement and adaptation to actual needs of the different sectors of employee activities at the campus USP in Ribeirão Preto

Keywords: Occupational Health. Work Safety. Quality of Life.

\section{INTRODUÇÃO}

\section{Desde o final do século XVII há uma preocupação com a saúde do trabalhador} relacionando os riscos e as doenças desenvolvidas. Em 1700, Ramazzini estabeleceu um verdadeiro tratado sobre doenças ocupacionais, sendo o primeiro documento na literatura mostrando a relação trabalho / doença. As perguntas a respeito da ocupação profissional das pessoas na anamnese clínica foram informações importantes para tratamentos específicos como também para elaborar medidas de prevenção. Apesar desse resultado, não há registro de intervenções na época [14].

A revolução industrial, na Inglaterra no início do século XVIII, trazia junto com a máquina o aumento significativo de acidentes relacionados ao trabalho, e das condições de insalubridade nos ambientes das fábricas. Foi graças à percepção coletiva de que o trabalho nessas condições estava levando danos à saúde, com adoecimento e morte, é que surgiu, no início do século XIX, a medicina do trabalho [20].

A expressão "serviço de medicina do trabalho" designa um serviço no local de trabalho ou próximo a este, organizado para assegurar a proteção aos trabalhadores com relação aos riscos a que estão expostos e das condições onde é desenvolvido esse trabalho, além de adequá-lo aos perfis e às aptidões, tendo como consequência o bem estar físico e mental. Com foco na proteção à saúde do trabalhador, a Organização Internacional do Trabalho (OIT) tornou-se uma referência sobre o assunto. No Brasil, a legislação trabalhista foi ampliada no Governo Vargas, com a Consolidação das Leis do Trabalho (CLT), mantendo sob controle do Estado as demandas sociais e trabalhistas, como também o Ministério do Trabalho, Indústria e Comércio, quando foi instituída a fiscalização do trabalho, somente efetivada muitos anos depois $[1,11,13]$.

As primeiras normas trabalhistas na Inglaterra (Lei de Saúde e Moral dos Aprendizes - 1802), com a anuência do Estado, objetivou a redução dos riscos ocupacionais. Entretanto, foi apenas com a medicina social, que foram reconhecidas as condições de trabalho como um dos aspectos importantes relacionados às condições de saúde e de vida, hoje, qualidade de vida no trabalho $[6,18]$.

A identificação de problemas relacionados à saúde do trabalhador no Brasil também foi registrada no século XIX, porém foi no século seguinte, com o desenvolvimento científico da medicina e a evolução tecnológica das áreas de engenharia e 
segurança do trabalho, incluindo-se a ergonomia, que se compôs um modelo da saúde do trabalhador, extensivo à saúde coletiva [5].

A expressão "medicina do trabalho", segundo a Recomendação 112 da OIT, define a prática nos locais de trabalho, com objetivo de proteção contra riscos, adaptação e adequação física e mental dos trabalhadores segundo suas aptidões, colaborando para elevar o bem-estar coletivo.

O enfoque de saúde ocupacional deu-se pela CLT (1970) pela obrigatoriedade de equipes técnicas multidisciplinares (Norma Regulamentadora 4), pela avaliação dos riscos ambientais (NR 15) e pelo acompanhamento das condições de saúde do trabalhador ( $\mathrm{NR}_{7}$ ), segundo a portaria 3214 / 78. Vale ressaltar que esse modelo estabelecido não atingiu seus objetivos, principalmente porque, apesar de focar a questão no coletivo, continuou a abordar o trabalhador como um "objeto" das ações de saúde. Deve-se considerar que existem profundos impactos psicológicos, sociais, financeiros e físicos que uma lesão ou doença no local de trabalho normalmente ocasiona [9].

A transição da saúde ocupacional para a saúde do trabalhador por meio da promoção da saúde é um processo ainda em andamento, considerado um caminho longo e com resultados não imediatos, uma vez que pauta-se pela educação, com objetivo definido de modificar o comportamento das pessoas e o dito "estilo de vida". Importante lembrar que saúde não é uma condição estável, está diretamente ligada e é influenciada pelo meio em que se vive e pode ser balizada pelo resultado do trabalho que cada um e o coletivo consegue adequar, segundo anseios e necessidades, inclusive de satisfação, considerando-se a complexidade das relações de saúde-trabalho $[7,10,8,2]$.

Ainda que sejam evidentes as dificuldades com relação aos métodos de ensino-aprendizagem, a chamada saúde do trabalhador pode-se dizer que busca uma explicação concreta sobre o adoecer, com relação à sua causa e o morrer como sua maior consequência, de um modo geral como também de forma particular, pelo estudo dos processos de trabalho, desenvolvimento e capacitação de recursos humanos, considerando valores como representações sociais, crenças e idéias, como o consumo de bens e serviços, visando agregar valor à vida das pessoas [22].

A Norma Regulamentadora 32 (NR-32) foi instituída pela portaria 485 , de 11 de novembro de 2005, com a finalidade de estabelecer as diretrizes básicas para implementação de medidas de proteção à segurança e à saúde dos trabalhadores dos serviços de saúde, bem como daqueles que exercem atividades de promoção de assistência à saúde em geral. Esta norma contempla entre seus itens, a capacitação dos trabalhadores antes do início das atividades e de forma continuada devendo ser ministrada sempre que ocorra uma mudança das condições de exposição dos trabalhadores aos agentes, durante sua jornada de trabalho e por profissionais de saúde familiarizados com os riscos inerentes aos agentes biológicos, químicos e físicos (radiações ionizantes).

É fato que nem todos os setores de saúde atendem de modo satisfatório e suficiente a NR-32, uma vez que na literatura nacional constam relatos de acidentes de trabalho $[16,21]$.

Considerando a necessidade de divulgação e implementação desta norma, fez-se a proposta à Pró-Reitoria de Cultura e Extensão da Universidade de São Paulo de um 
curso anual objetivando a capacitação dos servidores não docentes, visando segurança em seu desempenho profissional.

\section{MATERIAIS E MÉTODOS}

Considerando-se a área de saúde, a NR-32 - Segurança e Saúde no Trabalho em Serviços de Saúde foi a base para a implementação do curso Saúde e Segurança do Trabalho - capacitação segundo a NR-32, oferecido em 2010, 2011, 2012 e 2013, respectivamente.

O curso foi idealizado e montado pelas seguintes Unidades da Universidade de São Paulo do Campus de Ribeirão Preto: Odontologia, Medicina e Enfermagem com o apoio do Serviço Especializado em Engenharia de Segurança e Medicina do Trabalho (SESMT-RP).

Direcionado para os servidores não docentes, o curso teve como objetivo capacitá-los em segurança e higiene no trabalho, propiciando condições para a minimização dos riscos aos quais estão expostos durante o exercício de suas funções, considerando os diferentes agentes nos específicos ambientes laborais. Propiciar a aquisição de conhecimentos necessários sobre prevenção de acidentes e doenças ocupacionais além de orientar sobre normas vigentes e medidas de segurança no trabalho.

O curso, em todas as suas edições foi uma proposta para a Pró-Reitoria de Cultura e Extensão da USP, com as seguintes características:

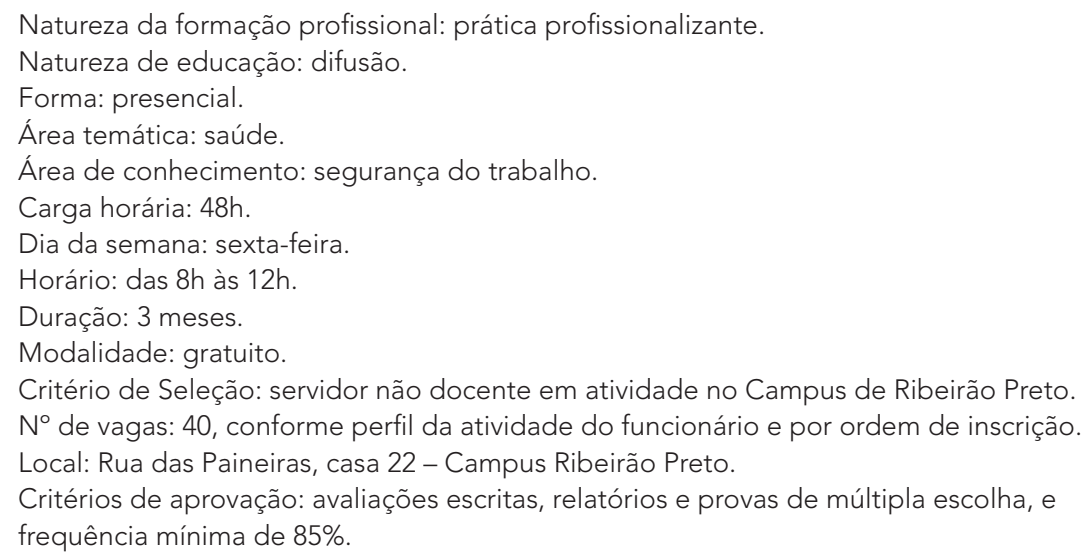

Os recursos físicos para a realização dos cursos foram disponibilizados na casa 22 da Rua das Paineiras - Campus Ribeirão Preto, denominada de Centro Multidisciplinar de Promoção à Saúde e Prevenção de Doenças. Conta com pessoal e infraestrutura própria, com recepção, sala de aula com equipamento de multimídia, acesso à internet, lousa didática e capacidade para 40 vagas. Além disso, possui espaço próprio para descanso nos intervalos das aulas / palestras, privilegiado pelo verde.

$\mathrm{O}$ curso foi oferecido anualmente e em todas as edições os alunos receberam, graças ao apoio financeiro da Pró-Reitoria de Cultura e Extensão da USP, uma pasta com os textos referentes a todas as palestras ministradas. Esse material foi preparado 
pelos palestrantes para possibilitar o estudo e facilitar a sua utilização pelos alunos em outros momentos durante o desenvolvimento de suas atribuições, mesmo depois da conclusão do curso. Este instrumento é importante para auxiliar o desenvolvimento de condutas preventivas de preservação à saúde e à qualidade de vida. Os alunos receberam também blocos para anotações e caneta esferográfica.

Os palestrantes foram especialmente convidados de acordo com suas especialidades e os com residência em outras cidades, receberam transporte e hospedagem.

O programa do curso foi atualizado ao longo desses anos, sendo composto atualmente por 17 títulos:

1. Meio Ambiente, Saúde e Desenvolvimento Sustentável

2. Organização do Trabalho/ Promoção da Saúde e Qualidade de Vida no Trabalho

3. Biossegurança

4. O processo Saúde/Doença - Imunização

5. Antissepsia das Mãos

6. Prevenção de Riscos Ambientais / Aspectos Legais (legislação nacional e internacional)

7. Grupos de Riscos / Mapa de Riscos

8. Medidas Gerais de Segurança e Proteção/Segurança na Manutenção de Máquinas e

Equipamentos

9. Manuseio e Descarte de Resíduos de Origem Biológica e Radioativa

10. Manuseio e Armazenamento Correto de Produtos Químicos

11. Conservação e Limpeza em Geral

12. Controle Médico de Saúde Ocupacional

13. Notificação e Registro de Acidentes

14. Noções de Prevenção de Incêndio

15. A importância da Ergonomia nas Atividades Profissionais para a Qualidade de Vida

16. Biossegurança: O Destino correto dos Resíduos Químicos

17. Plano de Gerenciamento de Resíduos do Serviço de Saúde - PGRSS

A divulgação dos cursos sempre foi realizada por meio de ofício oriundo do SESMT-RP, encaminhado às unidades do Campus USP de Ribeirão Preto, que os direcionava aos departamentos para conhecimento dos servidores. Os que demonstravam interesse com a anuência da chefia imediata tinham suas inscrições realizadas, conforme critérios já salientados, pelo preenchimento das fichas que acompanhavam os ofícios e retornavam à origem.

Os alunos foram avaliados por relatórios, provas escritas e testes de múltipla escolha, de cada assunto ministrado, observando-se frequência mínima de $85 \%$. Ao término de cada avaliação havia apresentação dos gabaritos, permitindo a discussão sobre o tema, e principalmente a possibilidade do aluno memorizar a resposta certa, melhorando seu nível de aprendizado real. A média final de cada aluno correspondeu à média aritmética. Aos que obtiveram médias e frequência mínima foi concedido certificado emitido pela Pró-Reitoria de Cultura e Extensão da USP. 


\section{RESULTADOS}

Os resultados estão expressos nas Tabelas 1 a 5 .

Tabela 1 - Avaliação do número de participantes por ano de realização do curso Saúde e Segurança do Trabalho - Capacitação segundo a NR-32 no Campus USP de Ribeirão Preto (2010-2013).

\begin{tabular}{cc}
\hline ANO & $\begin{array}{c}\text { NÚMERO DE } \\
\text { PARTICIPANTES }\end{array}$ \\
\hline 2010 & 42 \\
\hline 2011 & 33 \\
\hline 2012 & 36 \\
\hline 2013 & 28 \\
\hline TOTAL & 139 \\
\hline
\end{tabular}

Na Tabela 1 é evidenciado o número de participantes por ano de realização do curso. Nota-se que houve um decréscimo no número de participantes, sendo que em 2010 foram 42, enquanto que em 2013, 28.

Tabela 2 - Avaliação do número e percentual de participantes por gênero e ano de realização do curso Saúde e Segurança do Trabalho - Capacitação segundo a NR-32 no Campus USP de Ribeirão Preto (2010-2013).

\begin{tabular}{ccccccc}
\hline \multirow{2}{*}{ ANO } & \multicolumn{2}{c}{ HOMENS } & \multicolumn{2}{c}{ MULHERES } & \multicolumn{2}{c}{ TOTAL } \\
\cline { 2 - 7 } & N* & $\% *$ & N & $\%$ & N & $\%$ \\
\hline 2010 & 29 & 69,0 & 13 & 31,0 & 42 & 100,0 \\
\hline 2011 & 11 & 33,3 & 22 & 66,7 & 33 & 100,0 \\
\hline 2012 & 10 & 27,8 & 26 & 72,2 & 36 & 100,0 \\
\hline 2013 & 8 & 28,6 & 20 & 71,4 & 28 & 100,0 \\
\hline TOTAL & 58 & 100,0 & 81 & 100,0 & 139 & 100,0 \\
\hline
\end{tabular}

O predomínio do gênero feminino em todos os cursos ministrados é evidenciado na Tabela 2.

* n, número; \%, porcentagem. 
Tabela 3 - Avaliação do número de participantes por lotação em Unidade e ano de realização do curso Saúde e Segurança do Trabalho - Capacitação segundo a NR-32 no Campus USP de Ribeirão Preto (2010-2013).

\begin{tabular}{|c|c|c|c|c|c|}
\hline UNIDADE & 2010 & 2011 & 2012 & 2013 & TOTAL \\
\hline Faculdade de Medicina de Ribeirão Preto & 18 & 11 & 16 & 5 & 50 \\
\hline $\begin{array}{l}\text { Prefeitura da Universidade de São Paulo Campus } \\
\text { Ribeirão Preto }\end{array}$ & 11 & 3 & 0 & 1 & 15 \\
\hline $\begin{array}{l}\text { Faculdade de Ciências Farmacêuticas de } \\
\text { Ribeirão Preto }\end{array}$ & 5 & 0 & 8 & 7 & 20 \\
\hline Superintendência de Saúde & 4 & 2 & 0 & 4 & 10 \\
\hline Faculdade de Odontologia de Ribeirão Preto & 3 & 13 & 4 & 6 & 26 \\
\hline Superintendência de Assistência à Saúde & 1 & 0 & 1 & 0 & 2 \\
\hline $\begin{array}{c}\text { Faculdade de Filosofia, Ciências e Letras de } \\
\text { Ribeirão Preto }\end{array}$ & 0 & 2 & 2 & 3 & 7 \\
\hline $\begin{array}{l}\text { Escola de Educação Física e Esportes de } \\
\text { Ribeirão Preto }\end{array}$ & 0 & 1 & 1 & 0 & 2 \\
\hline Escola de Enfermagem de Ribeirão Preto & 0 & 1 & 3 & 2 & 6 \\
\hline Serviço de Verificação de Óbitos do Interior & 0 & 0 & 1 & 0 & 1 \\
\hline TOTAL & 42 & 33 & 36 & 28 & 139 \\
\hline
\end{tabular}

De acordo com a Tabela 3, as unidades com maior número de participantes no transcorrer dos cursos foram a Faculdade de Medicina de Ribeirão Preto, Faculdade de Odontologia de Ribeirão Preto e Faculdade de Ciências Farmacêuticas de Ribeirão Preto, respectivamente. 
Tabela 4 - Avaliação do número de participantes por local de atividade e ano de realização do curso Saúde e Segurança do Trabalho - Capacitação segundo a NR-32 no Campus USP de Ribeirão Preto (2010-2013).

\begin{tabular}{cccccc}
\hline LOCAL DE ATIVIDADE & 2010 & 2011 & 2012 & 2013 & TOTAL \\
\hline Biotério & 27 & 2 & 1 & 0 & 30 \\
\hline Laboratório & 8 & 18 & 29 & 22 & 77 \\
\hline SESMET* & 3 & 0 & 0 & 3 & 6 \\
\hline $\begin{array}{c}\text { Clínica de Atendimento } \\
\text { Odontológico }\end{array}$ & 1 & 5 & 1 & 1 & 8 \\
\hline Unidade Básica de Assistência à & 1 & 2 & 0 & 0 & 3 \\
\hline Saúde & 1 & 0 & 1 & 0 & 2 \\
\hline Creche (enfermaria) & 1 & 2 & 1 & 0 & 4 \\
\hline Bentro de Saúde Escola & 0 & 3 & 0 & 0 & 3 \\
\hline Administração & 0 & 1 & 0 & 2 & 3 \\
\hline Núcleo de Saúde Mental & 0 & 0 & 1 & 0 & 1 \\
\hline Enfermaria do Hospital & 0 & 0 & 1 & 0 & 1 \\
\hline Núcleo da Saúde da Família & 0 & 0 & 1 & 0 & 1 \\
\hline Total & 42 & 33 & 36 & 28 & 139 \\
\hline
\end{tabular}

Com relação ao local de atividade dos participantes por ano de realização do curso, em 2010 houve predomínio de funcionários provenientes de biotérios, e nos anos subsequentes de laboratórios.

\footnotetext{
* Serviço Especializado em Engenharia de Segurança e Medicina do Trabalho.
} 
Tabela 5 - Avaliação do número de participantes por função e ano de realização do curso Saúde e Segurança do Trabalho - Capacitação segundo a NR-32 no Campus USP de Ribeirão Preto (2010-2013).

\begin{tabular}{|c|c|c|c|c|c|}
\hline FUNÇÃO & 2010 & 2011 & 2012 & 2013 & TOTAL \\
\hline Técnico de Laboratório & 11 & 10 & 19 & 10 & 50 \\
\hline Auxiliar de Laboratório & 10 & 6 & 4 & 2 & 22 \\
\hline Auxiliar de Biotério & 7 & 0 & 0 & 0 & 7 \\
\hline Técnico de Biotério & 4 & 0 & 1 & 0 & 5 \\
\hline Técnico de Segurança do Trabalho & 3 & 0 & 0 & 0 & 3 \\
\hline Auxiliar de Consultório Dentário & 2 & 3 & 0 & 0 & 5 \\
\hline Auxiliar de Serviços Gerais & 2 & 2 & 0 & 0 & 4 \\
\hline Técnico de Enfermagem & 1 & 2 & 2 & 1 & 6 \\
\hline Especialista de Laboratório & 1 & 3 & 5 & 8 & 17 \\
\hline Auxiliar de Enfermagem & 1 & 0 & 0 & 0 & 1 \\
\hline Cirurgião-Dentista & 0 & 0 & 0 & 1 & 1 \\
\hline Médico & 0 & 0 & 0 & 1 & 1 \\
\hline Auxiliar de Saúde Bucal & 0 & 0 & 1 & 0 & 1 \\
\hline Químico & 0 & 0 & 0 & 2 & 2 \\
\hline Auxiliar de Materiais & 0 & 1 & 0 & 0 & 1 \\
\hline $\begin{array}{l}\text { Auxiliar/Técnico de Documentação e } \\
\text { Informação }\end{array}$ & 0 & 3 & 0 & 0 & 3 \\
\hline Auxiliar/Técnico de Assuntos Administrativa & 0 & 2 & 0 & 3 & 5 \\
\hline Técnico de Informática & 0 & 1 & 0 & 0 & 1 \\
\hline Farmacêutico & 0 & 0 & 1 & 0 & 1 \\
\hline Técnico de Nutrição e Dietética & 0 & 0 & 1 & 0 & 1 \\
\hline Enfermeiro & 0 & 0 & 1 & 0 & 1 \\
\hline Residente (fisioterapia) & 0 & 0 & 1 & 0 & 1 \\
\hline TOTAL & 42 & 33 & 36 & 28 & 139 \\
\hline
\end{tabular}

Ao longo das realizações dos cursos houve predomínio de técnicos e auxiliares de laboratório. Em 2010 as funções de técnico e auxiliar de biotério foram expressivas e em 2011 e 2013 nota-se a participação de servidores do setor administrativo (Tabela 5). 


\section{DISCUSSÃO}

Nos três primeiros anos foram realizadas inscrições via e-mail. No ano de 2013 o número de participantes foi menor devido ao fato que em 2012 houve um excedente de 80 inscritos e optou-se por contemplar, dentro da capacidade de vagas do curso, os funcionários com perfil compatível com a temática.

Como dito, a capacidade do local onde o curso é realizado é de 40 lugares. O primeiro curso mostrou que esta capacidade não poderia ser atingida em detrimento do conforto dos alunos, sendo assim, nos cursos subsequentes optou-se por limitar o número de participantes para 36.

De modo geral, a literatura demonstra o predomínio do gênero feminino nas áreas da saúde, que também foi constatado no nosso curso durante o período avaliado $[3,17,19]$.

Não causa estranheza o maior número de participantes (50/139) serem da Faculdade de Medicina de Ribeirão Preto, uma vez que esta é a Unidade com maior número de funcionários em atividade no campus, conforme estatísticas anuais da Prefeitura do Campus de Ribeirão Preto.

Embora a Faculdade de Odontologia de Ribeirão Preto seja uma Unidade com número menor de funcionários, quando comparado com as demais, notamos que o número de participantes foi expressivo (26/139).

O curso foi idealizado para capacitar funcionários com atividades em unidades de Saúde e dentro dessa proposta foi possível contemplar diferentes setores, destacando-se no período avaliado pessoal advindo de laboratórios e biotérios (107/139).

Todas as unidades de saúde do campus tiveram representações no período avaliado enviando funcionários que desempenham diferentes funções, o que vai de encontro ao intuito da organização do curso que era de atingir todos os níveis de progressão da carreira dos servidores não docentes. Fornecer ferramentas aos funcionários para o melhor desempenho das suas funções traz um aumento na qualidade do trabalho e da vida dessas pessoas o que, sem dúvida, reverte em maior produtividade no trabalho a ser desenvolvido [12].

No sistema de avaliação foi inserida a apresentação dos gabaritos após a realização das provas e discussões em grupos para melhor assimilação do conteúdo. Esse modelo limita o número de participantes, uma vez que o aproveitamento está diretamente relacionado à concentração no assunto, somente conseguido em menores grupos. Em função disso o número de servidores capacitados representa minoria frente ao universo do campus, porém espera-se que o aprendizado seja de grande valor no desenvolvimento das atividades.

Somos concordes com Cunha e Mauro [4], que salientam que é fundamental o despertar de consciência sobre a importância da NR-32 para a área de saúde e que deve ser construído com todos os atores.

\section{CONCLUSÃO}

O curso Saúde e Segurança do Trabalho - Capacitação segundo a NR-32 realizado no Campus USP de Ribeirão Preto, de 2010 a 2013, alcançou seus objetivos enquanto 
módulo de educação continuada. Decorrido quatro edições, os servidores capacitados representam minoria frente ao universo, sendo esta a principal limitação. No entanto, em função da característica do curso, considerando principalmente que alguns palestrantes oferecem prática demonstrativa, o grupo não pode ser muito numeroso, pois prejudicaria o ensino-aprendizagem. Como melhoria, nas próximas edições será inserida palestra com treinamento prático a respeito de noções de primeiros socorros. Ainda, almeja-se avaliar o impacto do curso nas unidades e serviços do campus, bem como a montagem de módulos avançados de capacitação, destinados aos alunos que já receberam a capacitação básica inicial, visando o aprimoramento e adequação às reais necessidades dos diferentes setores de atuação dos servidores do campus USP de Ribeirão Preto.

\section{REFERÊNCIAS}

[1] BRASIL. Decreto-lei no 5.452, de 10 de maio de 1943. Aprova a Consolidação das Leis do Trabalho. Diário Oficial [da] República Federativa do Brasil. Brasília, DF, 14 set. 2001, p.11.937.

[2] BRITO, J. C.; et al. Saúde, subjetividade e trabalho: o enfoque clínico e de gênero. Revista Brasileira de Saúde Ocupacional, São Paulo, v. 37, n. 3, p. 316-329, 2012. DOI: <http://dx.doi.org/10.1590/So303-76572012000200013>

[3] CANINI, S. R. M. S.; GIR, E.; MACHADO, A. A. Accidents with potentially hazardous biological material among workers in hospital supporting services. Revista Latino-americana de Enfermagem, Ribeirão Preto, v. 13, n. 4, p. 496500, 2005. DOI: <http://dx.doi.org/10.1590/So104-11692005000400006>

[4] CUNHA, A. C.; MAURO, M. Y. C. Educação continuada e a norma regulamentadora 32: utopia ou realidade na enfermagem? Revista Brasileira de Saúde Ocupacional, São Paulo, v.35, n.122, p. 305-313, 2010. DOI: <http:// dx.doi.org/10.1590/So303-76572010000200013>

[5] FAUSTO, B. Trabalho urbano e conflito social: 1890-1920. 3. ed. Rio de Janeiro: Difel, 1977.

[6] FRANÇA, A. C. L. Qualidade de vida no trabalho - QVT: conceitos e práticas nas empresas da sociedade pós-industrial. 2. ed. São Paulo: Atlas, 2004.

[7] GREEN, I. W.; KREUTER, L. W. Health promotion as a public health strategy for the 1990s. Annual Review of Public Health, v.11, p. 319-34, 1990.

[8] LANCMAN, S.; SZNELWAR, 1. I. Chistophe Dejours: da psicopatologia à psicodinâmica do trabalho. Brasília: Editora Fiocruz, 2004.

[9] LAX, M. B. Saúde ocupacional na região central do estado de Nova York: um ambulatório de doenças ocupacionais com financiamento público 25 anos depois. Revista Brasileira de Saúde Ocupacional, São Paulo, v. 38, n. 127, p. 149-161, 2013. DOI: <http://dx.doi.org/10.159o/So303-76572013000100016>

[10] MENDES, R. O impacto dos efeitos da ocupação sobre a saúde de trabalhadores. I - Morbidade. Revista de Saúde Pública, São Paulo, v. 22, n. 4, p. 311-326, 1988. 
DOI: <http://dx.doi.org/10.1590/Soo34-89101988000400007>

[11] MUNAKATA, K. A legislação trabalhista no Brasil. 2. ed. São Paulo: Brasiliense, 1984.

[12] CALDEIRA, T. A.; PEDROSO, R. Qualidade de vida no trabalho: diferentes percepções de um mesmo processo. Revista Olhar Científico, Ariquemes, v. 1, n. 1, p. 134-153, 2010.

[13] ORGANIZAÇÃO INTERNACIONAL DO TRABALHO. Recomendación sobre los servicios de medicina del trabajo en los lugares de empleo (Recomendación no 112 de la OIT adaptada en 24 de junio de 1959). In: Convenios y recomendaciones (1919-1966). Genebra, 1966. p. 1054-1058.

[14] PENA, P. G. L.; GOMES, A. R. A exploração do corpo no trabalho ao longo da história. In: VASCONCELLOS, L. C. F.; BARROS, M. H. Saúde, trabalho e direito: uma trajetória crítica e a crítica de uma trajetória. Rio de Janeiro: Educam, 2011. p. 85-124.

[15] RAMAZZINI, B. De morbis artificum diatriba, 1700. In: RAIMUNDOESTRELA, R, tradutor. São Paulo: Fundacentro, 2000.

[16] RIBEIRO, A. S.; et al. Caracterização de acidente com material perfurocortante e a percepção da equipe de enfermagem. Cogitare Enfermagem, Curitiba, v. 14, n. 4, p. 66o-666, 2009. DOI: <http://dx.doi.org/10.5380/ce.v14i4.16379>

[17] RISSI, M. R. R.; MACHADO, A. A.; FIGUEIREDO, M. A. C. Health care workers and Aids: A differential study about beliefs and affects associated with the experience of accidental exposure to blood. Cadernos de Saúde Pública, v.21, n.1, p.283-291, 2005. DOI: <http://dx.doi.org/10.1590/ So102-311X2005000100031>

[18] ROSEN, G. Da polícia médica à medicina social: ensaios sobre a história da assistência médica. Rio de Janeiro: Edições Graal, 1979.

[19] SCHILLIE, S.; et al. CENTERS FOR DISEASE CONTROL AND PREVENTION (CDC). VDV Guidance for Evaluating Health-Care Personnel for Hepatitis B Virus Ptrotection and Administering Postexposure Management. MMWR. Recommendations and Reports: Morbidity and Mortality Weekly Report. Recommendations and Reports / Centers for Disease Control, v. 62, n. RR-10, p 1-19, 2013.

[20] SCHILLING, R. S. F. Developments in occupational health. In: SCHILLING, R. S. F. (Ed.) Occupational health practice. 2. ed. London: Butherworths, 1981. p. 3-26.

[21] SIMÃO, S. A. F.; et al. Fatores associados aos acidentes biológicos entre profissionais de enfermagem. Cogitare Enfermagem, Curitiba, v. 15, n. 1, p. 87-91, 2010. DOI: <http://dx.doi.org/10.5380/ce.v15i1.17177>

[22] VILAS-BOAS, S. W. Apresentação do manual da gestão da Renast. In: ENCONTRO NACIONAL DA RENAST, 5., 2011. Relatório final. Brasília: Ministério da Saúde, 2011. 


\section{AGRADECIMENTOS}

À Pró-Reitoria de Cultura e Extensão da USP pelo apoio que viabilizou o oferecimento do curso em todas as suas edições.

Aos colaboradores, parceiros imprescindíveis na execução dos nossos cursos:

Adriano Menis Ferreira

Ana Paula de Tolvo Miranda

Ana Paula Macedo

Danilo Vitorino dos Santos

José Alcides Montes Sobrinho

Léa Mara Tosi Soussumi

Leonardo Monteiro Neves

Marco Antonio Cazarotti

Margareth de Lara Capurro Guimarães

Maria Rosa Rodrigues Rissi

Marisa de Cássia Registro Fonseca

Paulo Batista de Vasconcelos

Vânia Manna

Vera Lúcia Martinez Vieira

À Maria Amália Viesti Motta, secretária do Departamento de Odontologia Restauradora da Faculdade de Odontologia de Ribeirão Preto pelo auxílio e dedicação na inserção dos cursos no Sistema Apolo.

eVANDRo WATANABe professor doutor da Faculdade de Odontologia de Ribeirão Preto da Univerisdade de São Paulo (FORP-USP) - e-mail: evandrowatanabe@gmail.com

ANA MARIA RAZABONI professora associada da Faculdade de Odontologia de Ribeirão Preto da Universidade de São Paulo (FORP-USP)

ANGELA MARIA MAGOSSO TAKAYANAGUI professora associada da Escola de Enfermagem de Ribeirão Preto da Universidade de São Paulo (EERP-USP)

ALCYONE ARTIOLI MACHADO professora aposentada da Faculdade de Medicina de Ribeirão Preto da Universidade de São Paulo (FMRP-USP)

SANDRA MÁRCIA DE CASTRO engenheira de segurança do trabalho do Serviço Especializado em Engenharia de Segurança e Medicina do Trabalho de Ribeirão Preto da Universidade de São Paulo (SESMT-RP-USP) 